\title{
AN INTELLIGENT AND DATA DRIVEN MOBILE PLATFORM FOR EARLY CHILDHOOD DEVELOPMENT USING MACHINE LEARNING AND DATA MINING
}

\author{
Maggie Ding ${ }^{1}$ and Yu Sun ${ }^{2}$ \\ ${ }^{1}$ Orange County School of the Arts, Santa Ana, CA 92701 \\ ${ }^{2}$ California State Polytechnic University, Pomona, CA, 91768
}

\begin{abstract}
In China almost 96 million children live in rural areas. Some of these children suffer from malnutrition since parents or guardians do not have knowledge of nutritional plans or how to calculate nutritional values. However, most of the Chinese population has access to a mobile device. This paper proposes a mobile application, which runs on the IOS and Android platforms, to calculate nutritional values and recommend a nutritional menu. EZ Nutrition and Education is a mobile app that targets millions of parents and caregivers in rural China and provides a solution to the regions' early childhood underdevelopment problems. It provides recommendations for healthy meals and age-appropriate educational activities, measures children's daily intake of calories and macro-nutrients (protein, carbs, and fats), and provides a way to have fun through activities that teach skills and values so as to prevent the underdevelopment of rural children's physical, intellectual and mental growth. We applied our application to a group of participants (ages 6-12) and conducted a qualitative evaluation of the approach. The results show that the nutritional calculator feature can help parents improve the nutritional health of their children. Though two of the underweight participants lost weight and two of the overweight participants gained weight while using the recommended nutritional plan, we believe that the recommended nutritional menu could be an excellent feature for this application after we adjust some of the parameters since some parents claimed they could not follow the recommended menus.
\end{abstract}

\section{KEYWORDS}

Computer Science, Game, Art Design.

\section{INTRODUCTION}

In China, $40 \%$ of the 238 million children under the age of 14 live in rural areas. What happens to them matters deeply to China and the rest of the world. According to a Stanford study, one in three rural Chinese children are cognitively delayed and over half suffer from a lack of nutrition. [7] Of the 1,808 children in rural counties of the Shaanxi province (northwestern China) aged 12 to 30 months who were surveyed, 57\% scored below a certain threshold on an international infant mental development scale. "Research consistently shows that developmental and growth delays in this critical period before age 3 are irreversible, and can have serious implications even into adulthood." [8] Early childhood underdevelopment is dramatically changing the landscape of rural China, where almost 54\% of rural children live with some degree of language impairment. Many of these will go on to have difficulties learning at school.

David C. Wyld et al. (Eds): SOFEA, CTCM, BIBC, SIPR, NCWC, CSEN, EDTECH - 2021

pp. 187-204, 2021. CS \& IT - CSCP 2021

DOI: $10.5121 /$ csit.2021.111616 
There are two major issues with early childhood development (ECD) in rural China. First, studies show that "misinformation over childhood nutrition abounds in rural China. Parents do not know what kinds of food to feed their children at what age." [8] Proper food consumption is necessary to boost brain and body growth during pre-adolescence. Without healthy amounts of the right nutrients, the body and brain are unable to function as they should, leading to chronic illnesses and cognitive impairment. Secondly, researchers find rural caregivers, particularly grandparents, do not interact with their children enough. They do not know the benefits of early childhood activities. [9] They do not understand the importance of stimulating baby talk, and they do not respond to them and engage them in communication. They do not understand that the number of words babies are exposed to early on will determine the number of words they will be able to produce before age two. Also, caregivers do not know the importance of reading to babies. They do not understand that reading to babies opens up more learning opportunities. The fact that only $10 \%$ of rural children have more than five books means most rural children have reduced chances of developing an early, simulative imagination and may lack essential interpretive and communication skills. [10] Thirdly, caregivers do not play with their babies and they do not understand that babies learn important life skills through playing games.

Research has found "a troubling generational disconnect between the information-seeking behaviors and parenting practices of rural caregivers." [13] Millions of rural Chinese children suffer from misinformation about both education and nutrition during early childhood.

Parents in China care about child education. The problem is that there are not enough service providers in rural areas. However, state and private-sponsored early childhood development (ECD) centers are becoming more plentiful in urban areas. [14, 15] There are some ECD centers in rural areas, but they are often for children between the ages of three and six. ECD centers for children before age three are rare, and if there are any, they are usually too distant for rural families to take advantage of them. Living in sparse villages where families are busy and may be dependent on the economy makes wide-spread usage of ECD centers hard to achieve. Researchers have compared ECD centers to home-based intervention and found that the average impact of the center-based intervention was half that of home-visiting intervention. Although children benefit the most from ECD centers, rural children and their caregivers are less likely to participate in the programs. With this being the situation, it is hard to reach and educate rural parents or caregivers about nutrition and children's activities.

While apps and websites focusing on early childhood nutrition and education have been developed in China, they mostly target urban areas because they are more profitable. In rural areas, however, such as apps are unheard of.

We have developed an app that we believe will provide a solution to this growing problem. Mobile phones and apps are widely used in China. By November 2019, the number of mobile phone subscribers in China had skyrocketed to 1.6 billion. Close to 900 million people in China have accessed the internet via a mobile device, and our mobile app can be easily downloaded and accessed daily within rural homes.

To solve this nutrition deficiency problem, our app provides suggested food menus based on the user's profile, and the parent/caregiver is able to select these menus to use as references for what their children should eat throughout the week. Then the caregiver is able to record the amount and types of food their child or children consumes during the day. The system can calculate their daily intake of calories and macronutrients (protein, carbs, and fats). Users will then receive a rating for the day's meals, from bad to good, and recommendations to adjust the nutritional and/or caloric intake based on the data. The app updates these recommended menus as more 
meals are recorded, and users are able to navigate to any day or meal to see the specific information and rating for each item consumed.

To solve the issue of lack of interaction, the app also focuses on cognitive and mental development. The app offers a multitude of activities, such as playing hide-and-seek, listening to lullabies, and reading stories, which are all designed to engage children's minds. [11, 12] It also contains a built-in calendar, which not only gives caregivers foresight into the days ahead, but also allows them to become more organized in their daily activities.

Caregivers can easily navigate the system and find potential nutrients their children may lack or have too much of, as well as provide their children an alternative way to have fun through handpicked activities that teach them skills and values along the way.

To solve the early childhood underdevelopment problem, direct intervention through ECD centers, which is costly and less effective, is also limited. Our solution is unique - not only does it target the underprivileged, but it is also scalable. In addition to parents/caregivers, young kids and teenagers who have access to mobile phones can also use the app to monitor their own nutrition levels and participate in age-appropriate activities.

Compared to other well-known apps such as boohee.com, which aims to help adults control their weight, our app targets rural caregivers to help them learn to provide younger kids with a balance of nutrition and activities based on specific government guidance.

In this paper, we present an approach for parents, guardians, and teenagers to obtain access to recommended nutritional plans and calculate the total intake of calories, proteins, carbs and fat for themselves and their children. Since some Chinese parents, guardians, and teenagers do not have access to free nutritional plans and nutritional calculators, we propose a mobile application that runs on IOS or Android platforms to retrieve nutritional menus and calculate nutritional values. In two application scenarios, we demonstrate how the above combination of techniques improve children's health by calculating their daily calories and macronutrients (protein, carbs, and fats) and allow them to follow recommended nutritional menu plans. First, we demonstrated the usefulness of our approach via a comprehensive case study of the children's weight changes two weeks after their guardians recorded their daily body weight. Second, we analyzed the evolution of the children's weight during the two-week period in which they were using the recommended nutritional plan.

The rest of the paper is organized as follows: Section 2 provides details on the challenges that we met during the development period as well as some of the challenges faced by China's rural parents and their children; Section 3 focuses on the details of our solutions corresponding to the challenges mentioned in Section 2; Section 4 presents the relevant details of our experiment, followed by the related work in Section 5. Finally, Section 6 provides concluding remarks and points out future work for the project.

\section{Challenges}

In order to design a mobile application that provides recommendations for healthy meals and ageappropriate educational activities, measures children's daily intake of calories and macronutrients (protein, carbs, and fats), and provides a way to have fun through activities that teach skills and values, a few challenges have been identified as follows. 


\subsection{Challenge 1: Learning how to develop a nutritional mobile app for multiple platforms}

One challenge in developing this application was the cost in terms of time and knowledge. We wanted to make a nutritional app that could be available for both Android and Apple users. Because writing the app for both platforms would be time-consuming and require knowledge of at least two different languages, we had to consider software development that is suitable for both Android and Apple systems.

\subsection{Challenge 2: Chinese rural parents' access to tools}

How do China's rural parents get access to a tool that calculates daily calories, proteins, carbohydrates, and fats, as well as if their child is under, over, or of ideal weight and nutrition?

Chinese rural parents do not have access to tools that provide personal records of their children's diet or guidelines for a healthy diet. Rural parents might not have enough time or education to know if their children suffer from early childhood development problems. As a result, rural parents need a way to learn if their child suffers from malnutrition early so the child does not suffer other diseases in the future.

\subsection{Challenge 2: Chinese rural parents' access to nutritional plans and physical activities}

How do China's rural parents get access to nutritional plans and physical activities to improve their children's health?

In order to improve children's health and boost their immune systems, parents need to have adequate nutrition plans and have their children engage physical activities. However, many parents do not have access to free resources or the time to obtain nutritional plans or do healthy activities. We plan to provide a mobile application with which guardians can access nutritional menus and healthy activities that can boost their children's health.

\section{Solution}

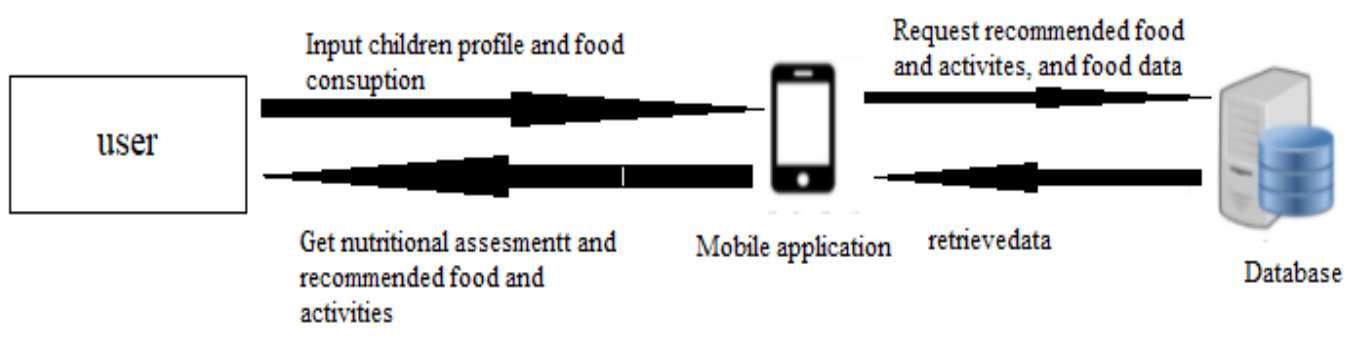

Figure 1. Overview of EZ Nutritional Education

To develop our mobile application (see Figure 1), we decided to use the Flutter software. [3] Flutter is an open-source UI software development kit developed by Google. This open-source UI provides the ability to develop applications for Android, IOS, Linux, Mac, Windows and web from a single codebase. The programming language that is used to code the Flutter app is Dart. Dart is a programming language developed by Google and is used to build servers and desktop 
applications. [5] By using Flutter we solved the issues mentioned in Challenge 1, so we could develop both IOS and Android apps using a single codebase.

For calories and macronutrient data (protein, carbs, and fats), we used information from the National Health Commission of the People's Republic of China to make the app suitable for all children. In order to calculate daily caloric intake as well as macronutrients (protein, carbs, and fats) and customize the information according to the user's requirements, we grouped users according to age, gender, and physical measurements such as height and weight.

For the total calories and macronutrients (protein, carbs, and fats) of each food, we used data provided by WebMD [6] and saved the data in our database. Similarly, we saved recommended food and activities in our database.

In order to use the mobile application, a user must enter his/her child's information, such as name, weight, height, age and gender. Then the system redirects to the home page. To get the recommended food, the system sends a request to the database, which retrieves the information. On the home page, the user can then see the recommended nutritional plans for breakfast, lunch and dinner or create his/her own nutritional plan. If the user decides to create a nutritional plan, they would select the meal time and add the food. After the user selects the food, the screen displays the calories and macronutrients (protein, carbs, and fats). Another functionality of the mobile app is that users can get personalized recommendations for activities according to their inputted data. Finally, the mobile app also allows users to edit their children's profile after it is created.

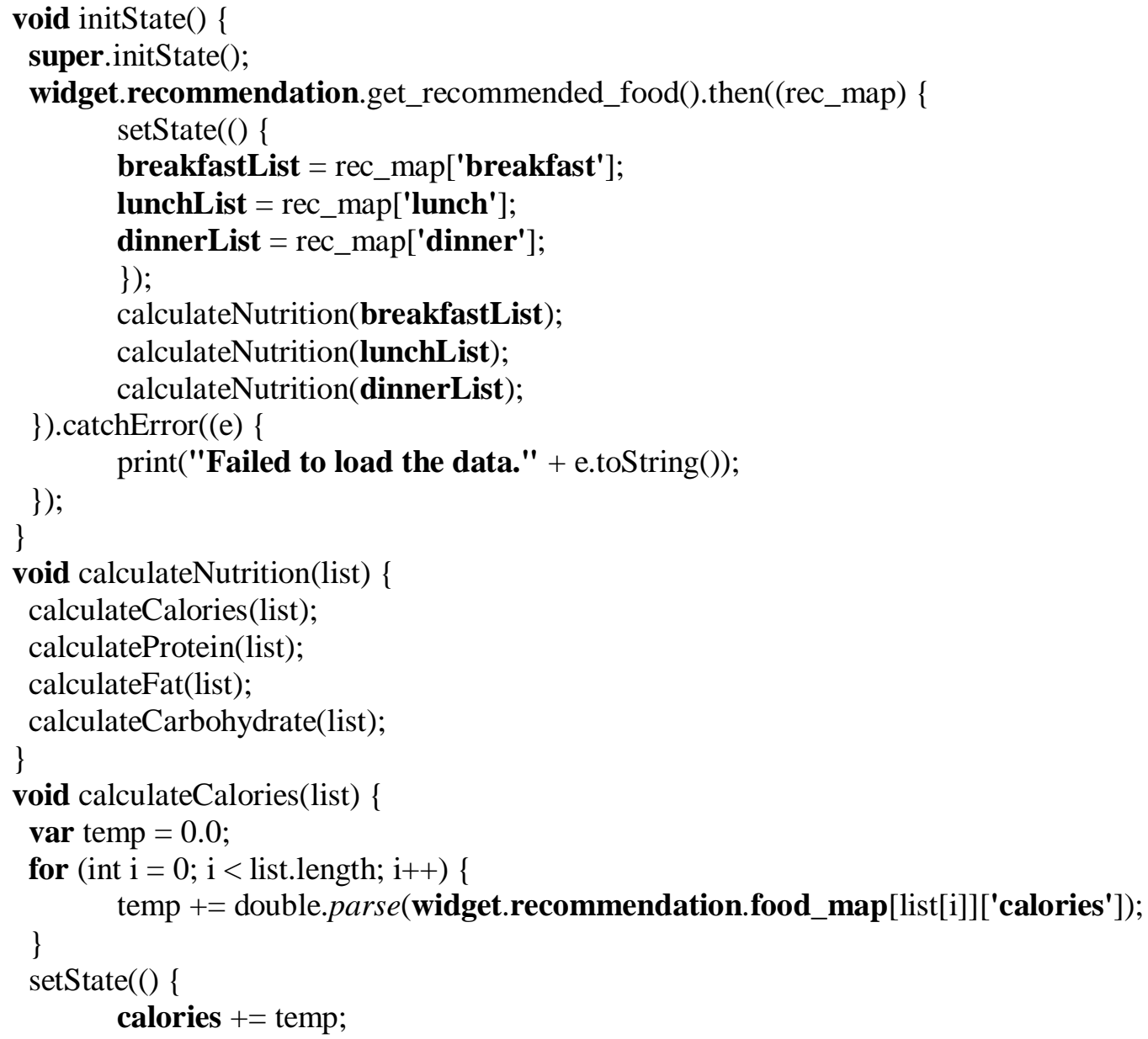




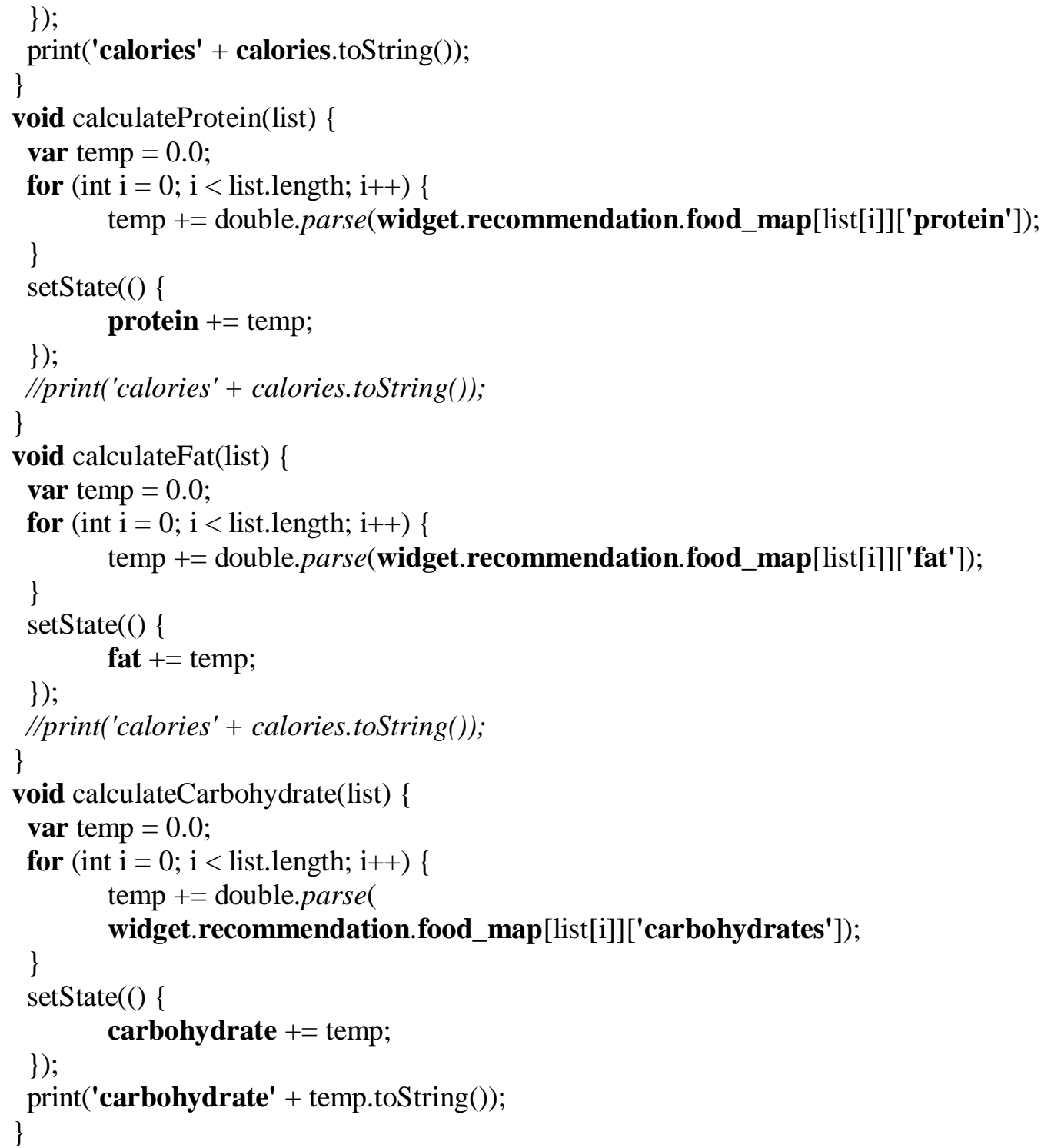

This page recommends food for users based on their personal data (see Figure 2). It also displays calories, proteins, fats, and carbohydrates in the recommended menu for users to see. We input the recommended data from the database. The database includes all data for the food. We coded to summarize data for users. 


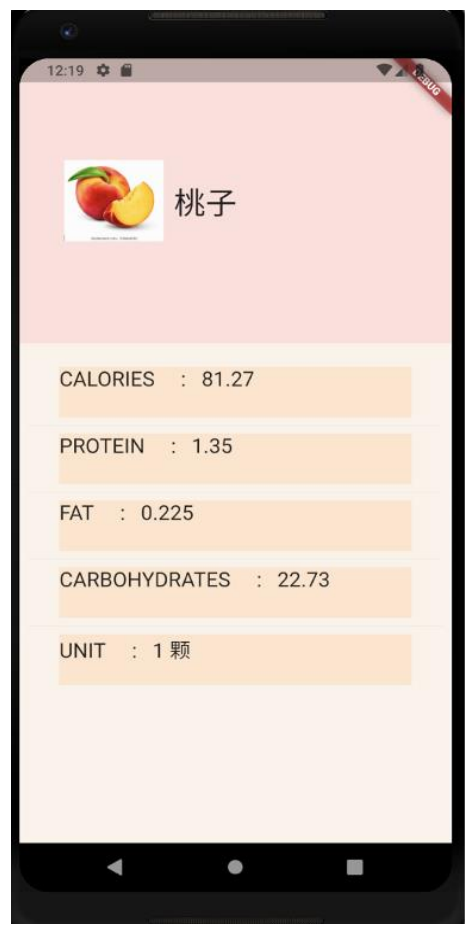

Figure 2. Recommending food

Expanded(

flex: 7 ,

child: Container(

margin: EdgeInsets.only(top: 15),

child: ListView.separated(

padding: const EdgeInsets.all(8),

itemCount: food_details.length,

itemBuilder: (BuildContext context, int index) \{

return Container(

margin: EdgeInsets.only(left: 30, right: 30),

height: 50 ,

color: Color.fromRGBO $(255,223,191,100)$,

child: Text(

food_details[index].toUpperCase ()$+$

' $\quad$ ' '

widget.recommendation.food_map[widget.foodId]

[food_details[index]],

textAlign: TextAlign.left,

style: TextStyle(fontSize: 20),

) ,

);

\} ,

separatorBuilder: (BuildContext context, int index) $\Rightarrow$ const Divider(),

$$
) \text {, }
$$


This page provides the details for each food (see Figure 3). It gets the data from another file and presents it.

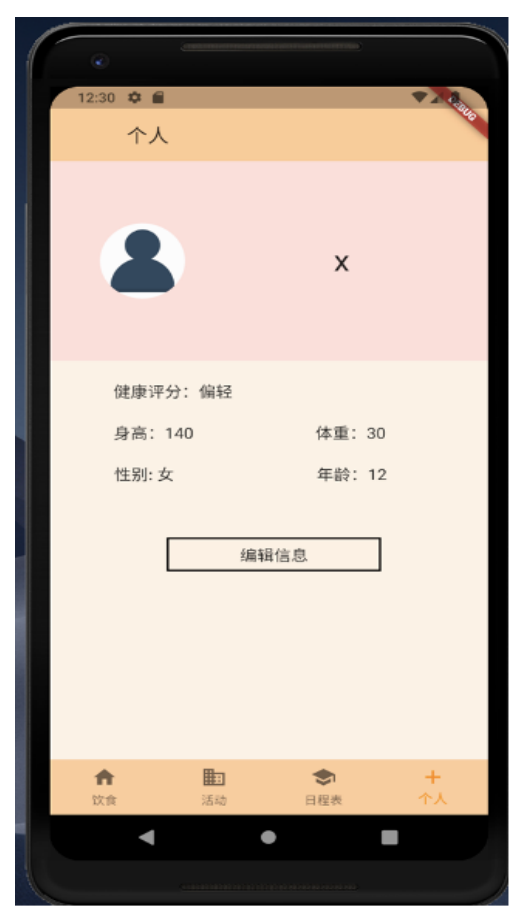

Figure 3. Details for each food

getHealthRating ()\{

var height $=$ double.parse(_height);

var weight $=$ double.parse(_weight);

if $($ gender $==$ 'Female' $)\{$

if (double.parse(_head) $<=2 \& \&$

double.parse(_height) $<=20 \& \&$

double.parse(_age) $<=2 \& \&$

double.parse(_weight) $<=30$ ) \{

healthRating = 'hum';

\}

if (height $<=106)$ \{

if (weight < 14) \{

healthRating $=$ '偏轻';

\} else if (weight $>17)\{$

healthRating = '偏重';

\} else \{

healthRating = '正常';

\}

\}

else if (height $<=108)$ \{

if (weight < 14.4) \{

healthRating = '偏轻';

\} else if (weight > 17.6) \{ 


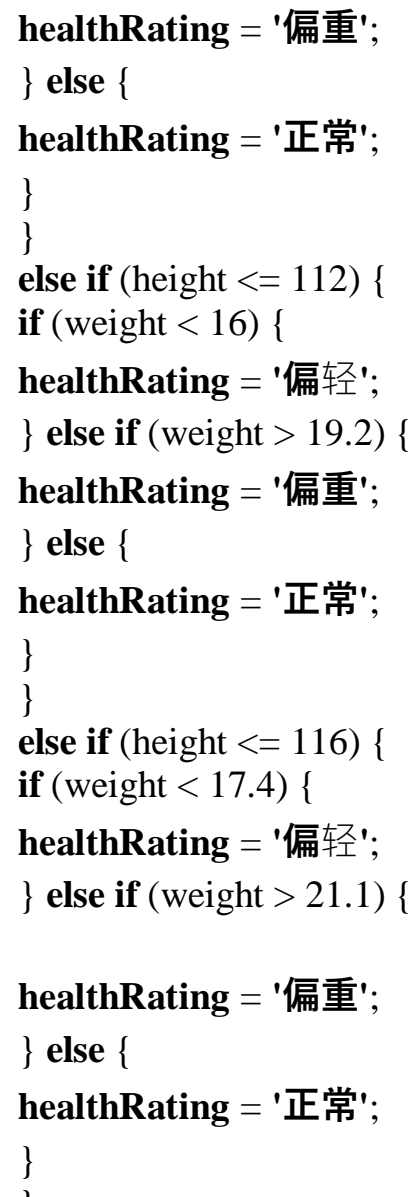

This page shows the user's data (see Figure 4). It also gives a rank for their body condition based on the data. We used an "if else" statement for this.

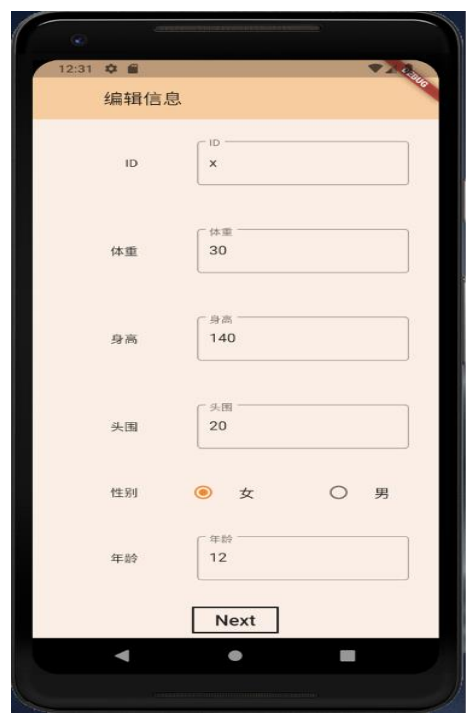

Figure 4. User's data and body condition

Widget build(BuildContext context) \{ 
return Scaffold(

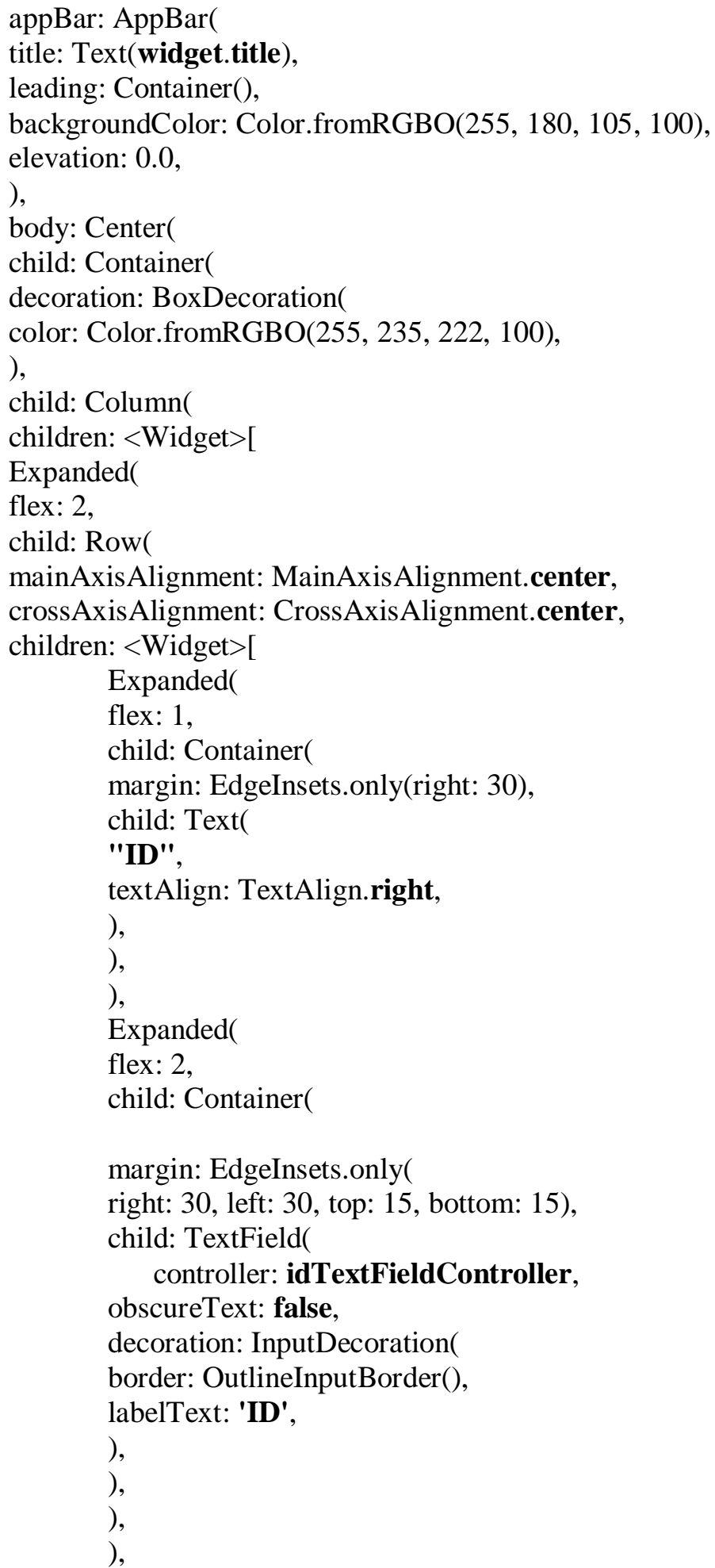

This page makes the user input their data for the app to use (see Figure 5). 


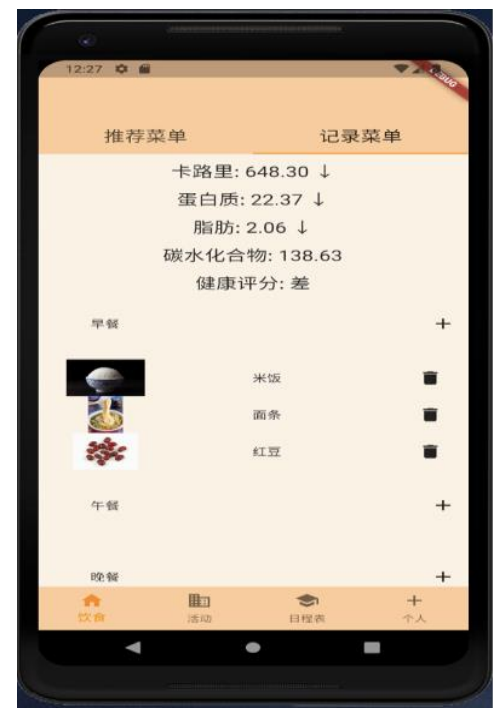

Figure 5. User inputting data

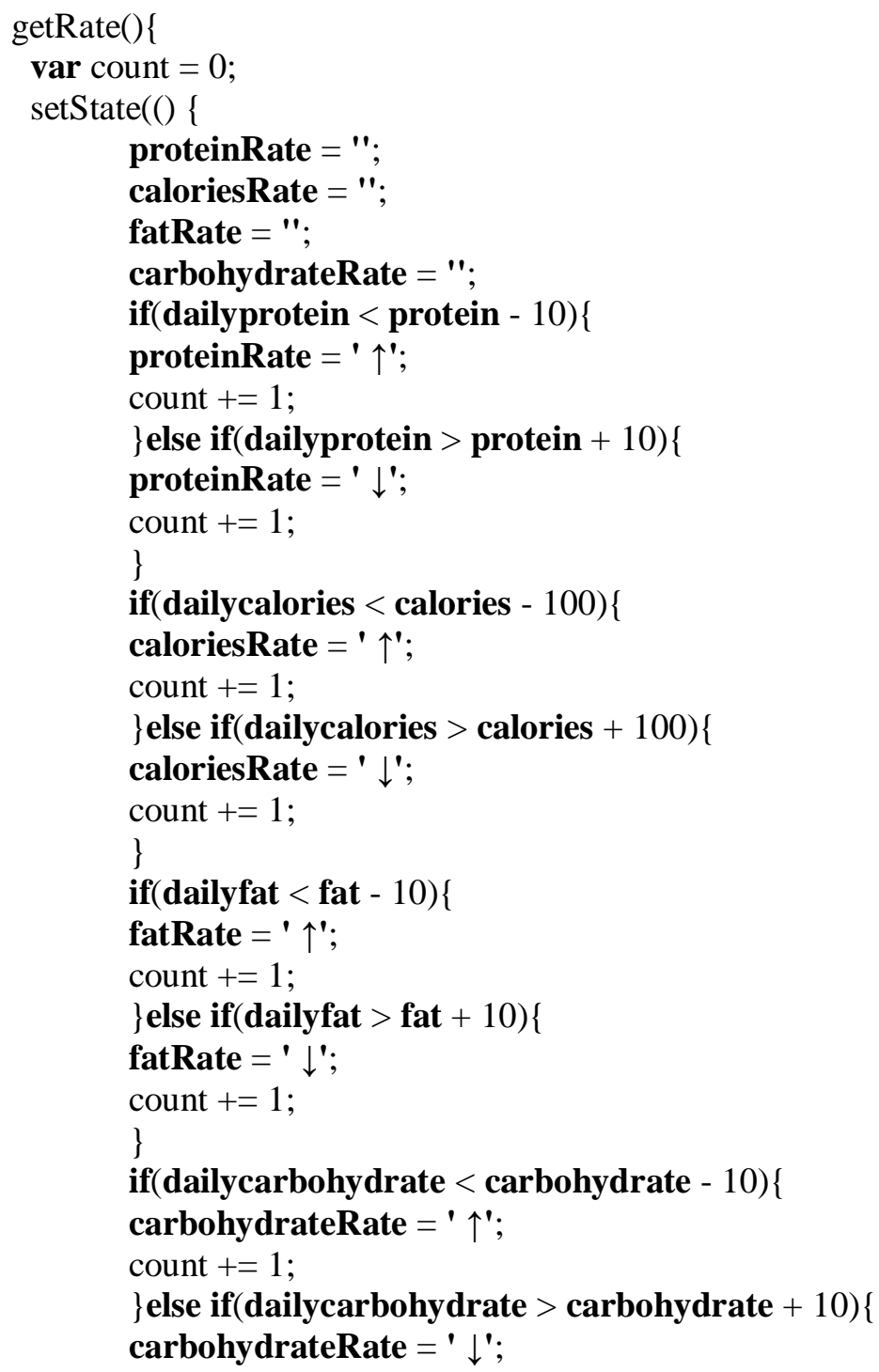




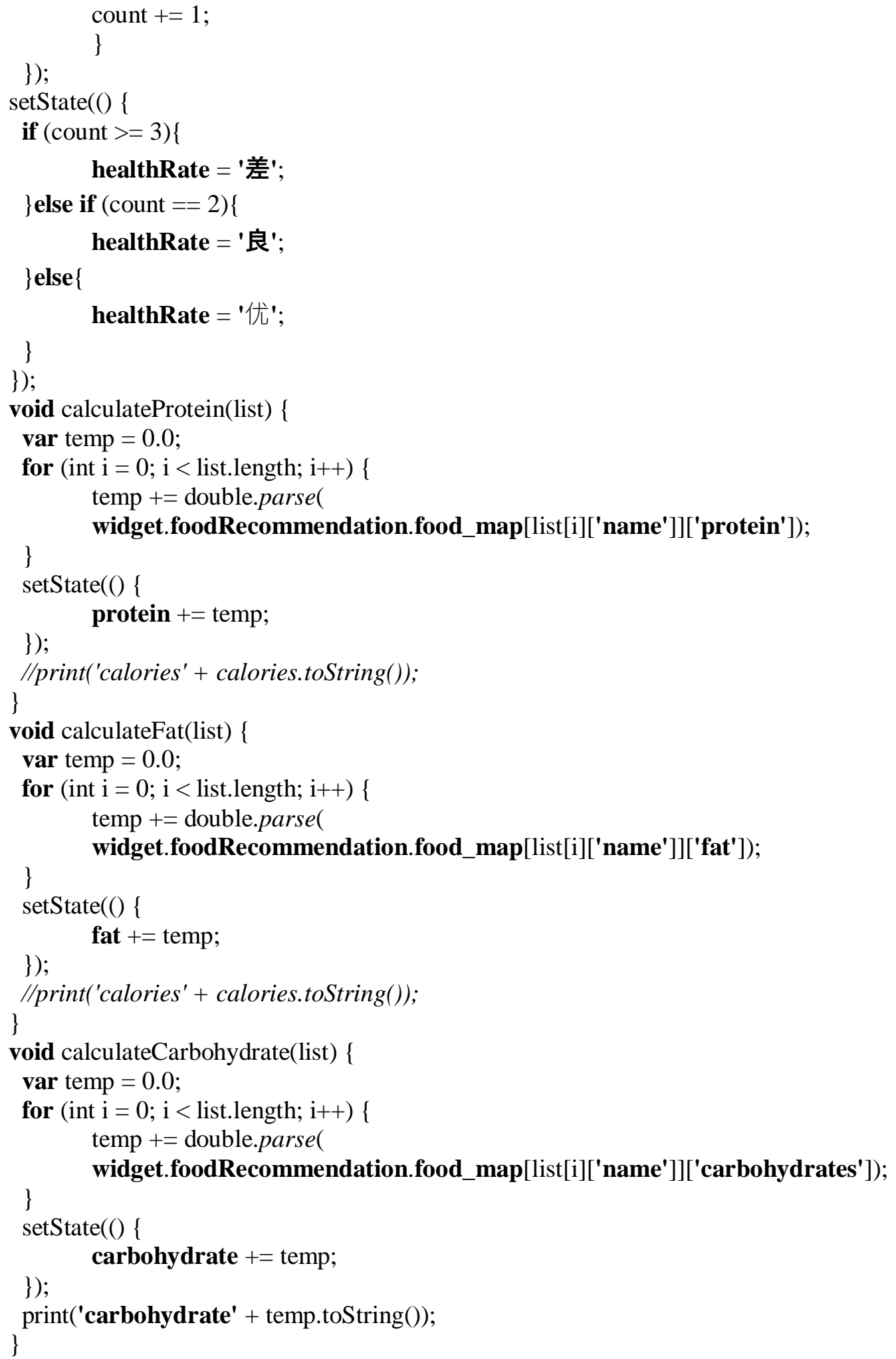

This page allows users to record their food and the app will give a rank based on their data. The program first gives a score for the recorded food based on the food and user data, then it gives a rank for the users based on the score. 
活动:

class_RecommendedActivityPageState extends State<RecommendedActivityPage $>\{$

void initState() \{

super.initState();

widget.recommendation.get_recommended_activities().then((rec_map) \{ setState(() \{

activityList $=$ rec_map;

//print(activityList);

\}$)$

\}).catchError((e) \{

I/print("Failed to load the data." + e.toString());

\}

\}$)$

var activityList $=[]$;

This provides recommended activities for the users.

活动detail :

Row(

children: <Widget $>[$

Expanded(

flex: 1 ,

child: Container(

margin: EdgeInsets.only(right: 30),

child: Text(

RecommendationActivity.activity_map[widget.activityid]

['description'],

textAlign: TextAlign.center,

) ,

)

)

],

),

This provides the detailed description of the recommended activities for the users.

Expanded(

flex: 2 ,

child: Container(

color: index $\% 2==0$

? Color.fromRGBO $(255,223,191,100)$

: Color.fromRGBO $(255,243,231,100)$,

height: height,

alignment: Alignment.center,

child: Text(

activityList [index]['time'],

textAlign: TextAlign.left,

) ,

)

)

Expanded(

flex: 1 ,

child: Container(

color: index $\% 2==1$

? Color.fromRGBO(255, 223, 191, 100) 
: Color.fromRGBO $(255,243,231,100)$, height: height,

width: 80 ,

alignment: Alignment.center,

child: Container(

height: height -30.0 ,

child: Image.network(

RecommendationActivity.activity_map [

activityList[index]['name']]['image']),

) ,

)

)

Expanded(

flex: 2 ,

child: Container(

color: index $\% 2==1$

? Color.fromRGBO $(255,223,191,100)$

: Color.fromRGBO $(255,243,231,100)$,

margin: EdgeInsets.only(right: 30),

height: height,

alignment: Alignment.center,

child: Text(

RecommendationActivity.activity_map [

activityList[index]['name']]['name'],

textAlign: TextAlign.left,

) ,

)

)

This provides the recommended schedule for users.

\section{EXPERIMENT}

To evaluate our approaches, we collected body weight fluctuation data for 16 participants for two weeks. These participants were from 6 to 12 years of age. Some participants suffered from being underweight or overweight, while others had a healthy weight. We conducted two different analyses to verify the two aspects of our approach: 8 participants that calculated their intake of calories, macronutrients (protein, carbs, and fats) and observed their weight changes, and 8 participants who used the recommendation nutritional plan and observed their weight after two weeks.

In this experiment, we used eight participants (6-12 years of age): four children who were overweight, two children who were underweight, and two children who had a healthy weight. We utilized the weight data of these participants, which was recorded by their guardians every day. First, the guardians inputted the information for their child into the EZ Nutrition and Education app. Then they included the food that they consumed in the corresponding mealtime every day. After two weeks, we collected the data and observed the weight of the first and last day of the child while using the mobile app. 


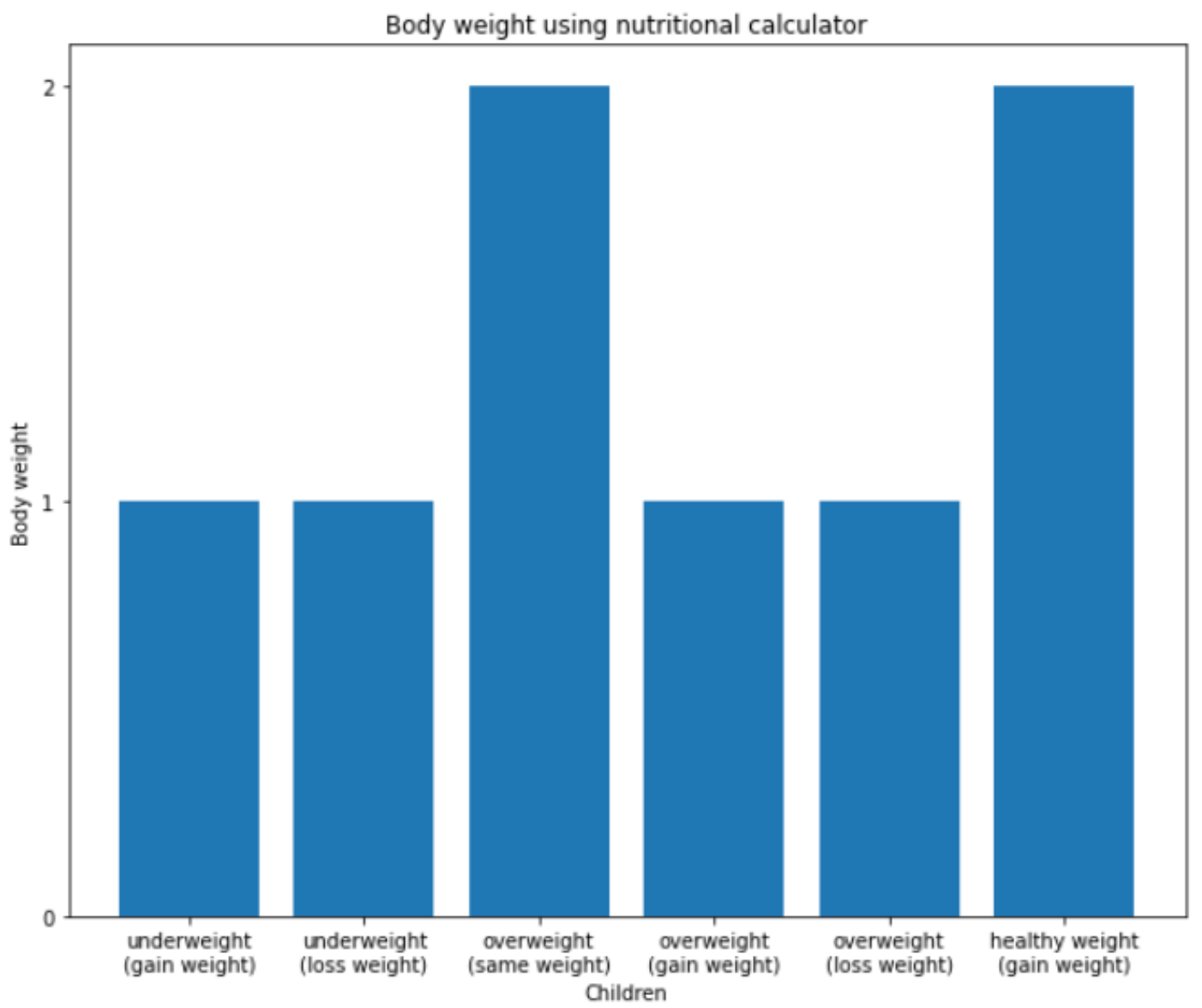

Figure 6. Bodyweights

As seen in Figure 6, some children lost weight, while others gained or maintained the same weight after two weeks. Some gained or lost 2-5 ounces of their bodyweight. We observed that one of the underweight children gained weight, while the other underweight child lost weight. For the four overweight children, we observed that two maintained the same weight, while one lost weight and the other gained weight. For the two children with healthy weight, both had gained weight after two weeks.

In the second experiment, similar to the previous one, we used eight participants (6-12 years of age): two overweight children, three underweight children, and three children with a healthy weight. We utilized the weight data that was recorded by their guardians. The guardians created their child's profile and followed the recommended nutritional menus for each corresponding meal time. After two weeks, we collected the data and noted the weight of children on the first and last days of using the recommended nutritional menus recommended by the app. 


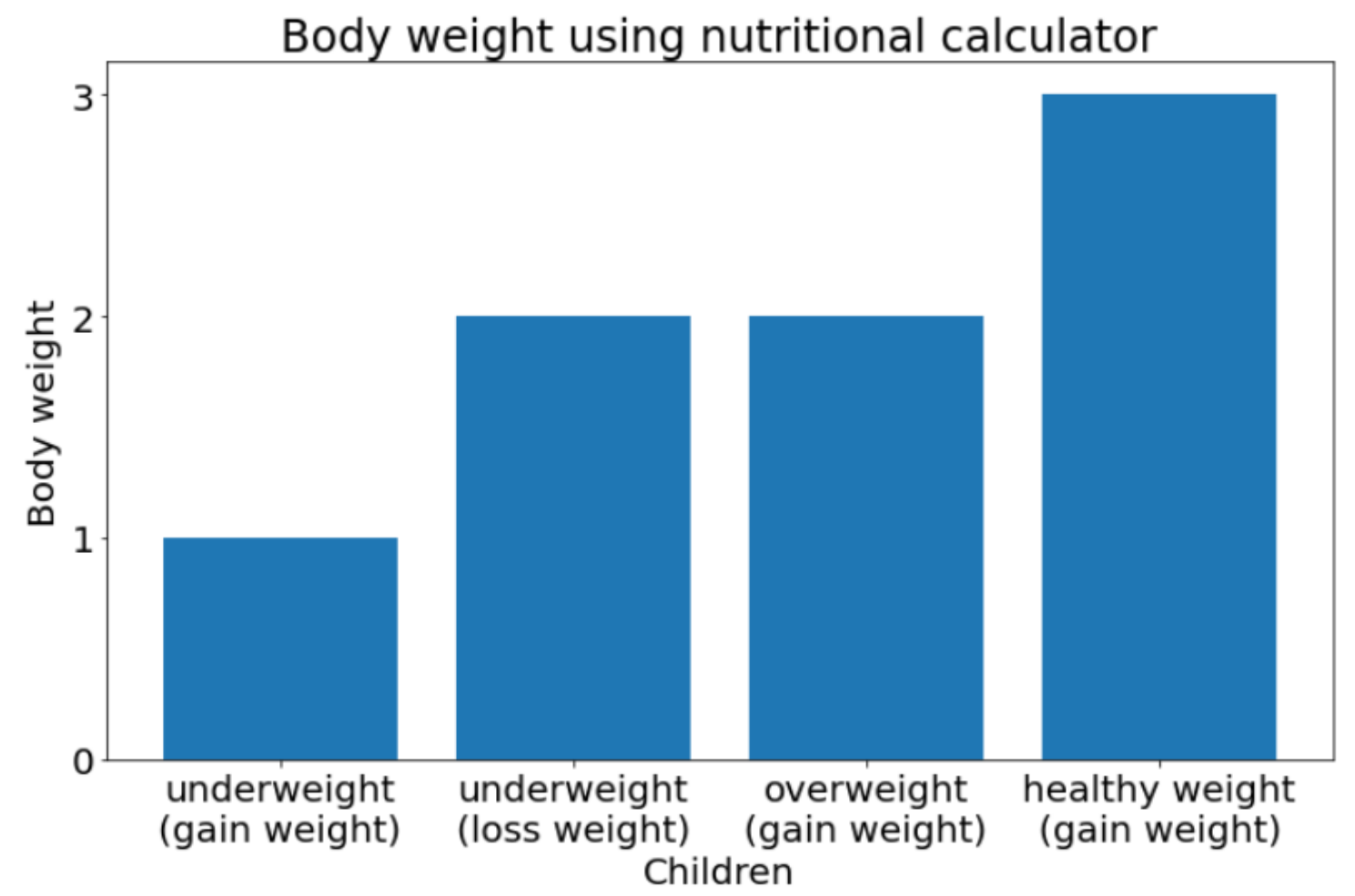

Figure 7. Bodyweights

Similar to the previous experiment, the results of this experiment were that some children lost weight while others gained after using the recommended nutritional menus (see Figure 7). Some gained or lost 2-8 ounces of their bodyweight. We observed that one child who was underweight gained weight, while the others lost weight. For the overweight children, we observed that both gained weight. For the children who had a healthy weight, we observed that all gained weight after two weeks.

Experiment 1 shows that the nutritional calculator feature can help parents to improve the nutritional health of their children. Even though we observed that one of the underweight participants lost weight and one of the overweight participants gained weight, we can claim that calculating the daily intake of calories and macronutrients (protein, carbs, and fats) can improve a child's nutritional health, overall. We do not know if parents followed the indicators that were displayed for their children since they did not record the daily intake of calories or macronutrients (protein, carbs, and fats) during the experiment.

Experiment 2 shows that some children gained or lost weight. Though two of the underweight participants lost weight and two of the overweight participants gained weight, we believe that the recommended nutritional menus might be an excellent feature for this application after we adjust some of the parameters, since some parents claimed that they could not follow the recommended menus. The reason was because some participants had food allergies or did not like the recommended foods.

\section{RELATED WORK}

Tine, F. et al. developed a mobile health application called Mhealth to manage acute malnutrition in five different countries: Afghanistan, Chad, Kenya, Mali and Niger. [1] Mhealth is a step-by- 
step guideline that helps health workers perform assessments and/or provide treatment for children that visit the CMAM program. It is built on the open-source Open Data Kit (ODK) platform, which provides feedback, saves medical history, and calculates z-scores. Unlike Mhealth, the objective of the EZ Nutrition and Education application is for parents to prevent malnutrition in their children, since they can build healthy diet plans according to their children's age and weight.

Dr. Aniruddha Vidwans, MD, a neonatologist, developed an iPhone app called NICU Nutrition Calculator. [2] The NICU Nutrition Calculator is a mobile app that assists health workers when they need to provide an adequate diet for premature infants, especially those weighing less than $1000 \mathrm{~g}$. It analyzes diverse resources for both enteral nutrition (EN) and parenteral nutrition (PN) to calculate the total calories/kilogram/day $(\mathrm{kcal} / \mathrm{kg} / \mathrm{d})$ and glucose infusion rates based on the diet prescription entered. The NICU Nutrition Calculator and our EZ Nutritional and Education app both calculate the daily intake of calories, however, our EZ Nutritional and Education app also calculates the daily intake of macronutrients (protein, carbs, and fats). The NICU Nutrition Calculator is developed only for the IOS platform, while our EZ Nutrition and Education app is developed for both Android and IOS devices. Another difference is that our EZ Nutritional Education app was developed for young children and teenagers, while the NICU Nutrition Calculator was developed for premature infants.

Lerena, L. et al. proposed an adaptive food recommendation system based on the user's profile and requirements. [3] The goals for their study and ours are similar since we also wish to develop software that can recommend nutritional plans depending on a user's IBM. However, we are focused on children and teenagers who might suffer from malnutrition.

\section{CONCLUSION AND FUtURE WORK}

In China, almost half of the population's children live in rural areas. Some of these children suffer from malnutrition since they do not have access to nutritional tools. In this paper, we proposed a mobile application that runs on IOS and Android platforms and can calculate the daily intake of calories, proteins, carbs and fats, as well as recommend nutritional menus. We developed the EZ Nutritional and Education app, which is a personal app for children according to their height, weight, gender, and age. The app can also recommend a nutritional plan and calculate the total calories, protein, carbs and fats that children consume each day. For our approach, we performed two experiments to observe and analyze the performance of the mobile features. We selected 16 participants (6-12 years of age), who used two different features of our mobile app, and recorded their bodyweights before and after a two-week period of using the app. Through our experiments, we observed that some children maintained the same weight, while others lost or gained weight after using the recommended nutritional menus and calculating their nutritional values. Some gained or lost 2-8 ounces of their bodyweight. Even though we observed that some of the underweight participants lost weight, and some of the overweight participants gained weight, we can claim that calculating the daily intake of calories, macronutrients (protein, carbs, and fats) can still improve children's nutritional health, ovrall. We believe that the recommended nutritional menu will still be an excellent feature for this mobile application after adjusting some of its parameters.

\section{REFERENCES}

[1] Van Willigen, Marieke. "Differential benefits of volunteering across the life course." The Journals of Gerontology Series B: Psychological Sciences and Social Sciences 55.5 (2000): S308-S318.

[2] Morrow-Howell, Nancy, Song-Iee Hong, and Fengyan Tang. "Who benefits from volunteering? Variations in perceived benefits." The Gerontologist 49.1 (2009): 91-102. 
[3] Wilson, John. "Volunteering." Annual review of sociology 26.1 (2000): 215-240.

[4] Michie, Donald, David J. Spiegelhalter, and C. C. Taylor. "Machine learning." Neural and Statistical Classification 13.1994 (1994): 1-298.

[5] Pedregosa, Fabian, et al. "Scikit-learn: Machine learning in Python." the Journal of Machine Learning Research 12 (2011): 2825-2830.

[6] Bracha, Gilad. The Dart programming language. Addison-Wesley Professional, 2015.

[7] Wu, Wenhao. "React Native vs Flutter, Cross-platforms mobile application frameworks." (2018).

[8] Moroney, Laurence, Moroney, and Anglin. Definitive Guide to Firebase. Apress, 2017.

[9] Peng, Dunlu, Lidong Cao, and Wenjie Xu. "Using JSON for data exchanging in web service applications." Journal of Computational Information Systems 7.16 (2011): 5883-5890.

[10] Stukas, A. A., Worth, K. A., Clary, E. G., \& Snyder, M. (2009). The Matching of Motivations to Affordances in the Volunteer Environment: An Index for Assessing the Impact of Multiple Matches on Volunteer Outcomes. Nonprofit and Voluntary Sector Quarterly, 38(1), 5-28. https://doi.org/10.1177/0899764008314810

[11] Finkelstein, Marcia A., Louis A. Penner, and Michael T. Brannick. "Motive, role identity, and prosocial personality as predictors of volunteer activity." Social Behavior and Personality: an international journal 33.4 (2005): 403-418.

[12] Davis, M. H., Hall, J. A., \& Meyer, M. (2003). The First Year: Influences on the Satisfaction, Involvement, and Persistence of New Community Volunteers. Personality and Social Psychology Bulletin, 29(2), 248-260. https://doi.org/10.1177/0146167202239050

[13] Segal, Mark R. "Machine learning benchmarks and random forest regression." (2004).

[14] Bunea, Florentina, Alexandre B. Tsybakov, and Marten H. Wegkamp. "Aggregation for Gaussian regression." The Annals of Statistics 35.4 (2007): 1674-1697.

[15] Kotsiantis, Sotiris B., I. Zaharakis, and P. Pintelas. "Supervised machine learning: A review of classification techniques." Emerging artificial intelligence applications in computer engineering 160.1 (2007): 3-24.

(C) 2021 By AIRCC Publishing Corporation. This article is published under the Creative Commons Attribution (CC BY) license. 